\title{
Internet das Coisas e Aprendizagem Colaborativa: Uma Revisão Sistemática da Literatura
}

\author{
Álvaro Maciel Cabral Dantas ${ }^{1}$, Hellan Dellamycow Gomes Viana ${ }^{1}$, \\ Jauberth Weyll Abijaude ${ }^{1}$, Péricles de Lima Sobreira ${ }^{1}$
}

\author{
${ }^{1}$ Universidade Estadual de Santa Cruz (UESC) \\ Rodovia Jorge Amado, Km 16, Salobrinho - 45662-900 - Ilhéus - BA - Brasil \\ \{alvaromcd, dellamycow\}@gmail.com, \{jauberth,plsobreira\}@uesc.br
}

\begin{abstract}
This work presents a Systematic Literature Review on the relationship between on the Internet of Things and Education with Collaborative Learning from the search of articles and studies, in four scientific databases, approaching the following research question: "What solutions, created in the last 10 years, have practically applied the Internet of Things in Education with Collaborative Learning?"following a defined methodology, it has found 33 primary studies that were analyzed and discussed.
\end{abstract}

Resumo. Este trabalho apresenta uma Revisão Sistemática da Literatura sobre a relação entre a Internet das Coisas e a Educação com Aprendizagem Colaborativa a partir da busca de artigos e estudos, em quatro bases de dados científicos, obedecendo a seguinte questão de pesquisa: "Quais soluções, criadas nos últimos 10 anos, aplicam na prática o conceito de Internet das Coisas na educação com Aprendizagem Colaborativa?”. Seguindo uma metodologia definida, encontrou-se 33 estudos finais que foram analisados e discutidos.

\section{Introdução}

A Internet é parte fundamental da sociedade e, sem dúvida, representa uma das maiores revoluções tecnológicas da história da humanidade. O conceito de que todas as coisas poderiam se comunicar com pessoas e aplicações é uma realidade, surgindo no final da década de 90 o conceito da Internet das Coisas (do inglês, Internet of things, ou IoT). A IoT se destaca pela sua versatilidade em inovar situações nos mais diversos campos, desde simples aplicações utilizadas no cotidiano das pessoas, até na sofisticada área aeroespacial [Mancini 2017]. Na educação, em particular para este estudo ao se considerar a Aprendizagem Colaborativa, a IoT também se faz presente.

A forma colaborativa de aprendizagem é um recurso educacional definido como a inserção de métodos para que alunos interajam entre si ou em conjunto com o professor, para a realização de atividades que aprimoram o conhecimento sobre um determinado assunto. A tecnologia é um dos principais suportes da aprendizagem colaborativa. A Internet, por exemplo, traz recursos como ambientes virtuais de aprendizagem, fóruns, wikis e outras ferramentas que concentram a discussão e colaboração de diversas pessoas sobre um tema em específico.

À união do aprendizado colaborativo com a tecnologia dá-se o nome de Aprendizagem Colaborativa com Suporte Computacional (do inglês Computer-Supported Collaborative Learning, ou CSCL). A CSCL surgiu durante a década de 1990, opondo-se 
VII Congresso Brasileiro de Informática na Educação (CBIE 2018)

Anais do XXIX Simpósio Brasileiro de Informática na Educação (SBIE 2018)

às ferramentas computacionais que restringiam os alunos à aprendizagem de forma individual. A sua definição básica pode ser dada como um campo educacional onde se estuda a maneira em que as pessoas podem aprender em grupo com a ajuda de recursos tecnológicos/computacionais.

Observando-se esta tendência, em 2017 foi iniciada, na Universidade Estadual de Santa Cruz, uma pesquisa que utiliza tecnologias de IoT com estudantes do ensino médio, em um cenário colaborativo, para a aprendizagem do conceito de programação. Este projeto motivou a elaboração desta Revisão Sistemática, cuja descrição se encontra organizada com descrito a seguir: a seção 2 discute os procedimentos metodológicos, apresentando as etapas que caracterizam a revisão; a seção 3 discute as tecnologias utilizadas nos estudos encontrados; a seção 4 analisa os resultados obtidos, através de tabela e gráficos, e, por fim; a seção 5 apresenta as considerações finais da pesquisa.

\section{Procedimentos Metodológicos}

Segundo [Kitchenham and Visaggio 2007], uma Revisão Sistemática (Systematic Literature Review - SLR, em inglês) é um estudo secundário que usa uma metodologia bemdefinida e confiável para identificar, analisar e interpretar os estudos que estejam disponíveis e que sejam relevantes a uma questão de pesquisa particular. Ela pode ser realizada com dois objetivos distintos: identificar lacunas existentes em uma área de pesquisa, ou fornecer um conjunto relevante de trabalhos relacionados para embasar novas pesquisas. Nesta seção são apresentados os detalhes mais importantes do processo metodológico desta Revisão Sistemática.

\subsection{Intervalo de Tempo para Pesquisa}

Do fim da década de 1990, quando o termo “Internet das Coisas"surgiu, até 2018, essa tecnologia tem se desenvolvido de forma acelerada [Li et al. 2018]. Nesta SLR, considerouse estudos realizados apenas no período dos últimos 10 anos, compreendido entre 2009 e (maio de) 2018, por considerar-se que, por razões tecnológicas, seja mínimo o número de trabalhos práticos que relacionam diretamente IoT e CSCL antes do ano de 2009.

\subsection{Objetivo e Questão de Pesquisa}

O objetivo desta SLR é identificar, analisar e interpretar como as tecnologias relacionadas à IoT são aplicadas na educação com Aprendizagem Colaborativa. Tendo em vista o dever da questão de pesquisa em exemplificar os objetivos do estudo, elaborou-se a seguinte questão: “Quais soluções, criadas nos últimos 10 anos, aplicam na prática o conceito de Internet das Coisas na educação com aprendizagem colaborativa?"

\subsection{Definição das Palavras-chave}

A definição das palavras-chave em SLRs é feita a partir das questões de pesquisa sob análise. Inicialmente realizou-se testes com palavras que seriam utilizadas como palavraschave nas buscas. Concluiu-se que as palavras deveriam ser todas no idioma inglês e que alguns sinônimos de "Internet of Things"deveriam ser usados pelo fato que determinados pesquisadores da área da educação utilizam o conceito de IoT, sem identificar tal conceito. Assim, foram consideradas as seguintes combinações: "Computer-Supported Collaborative Learning"e "Internet of Things"; "Computer-Supported Collaborative Learning"e "IoT"; "Collaborative Learning"e "Internet of Things"; "CSCL"e "Internet of Things"; "CSCL"e "IoT"; "CSCL"e "Sensor"; "CSCL"e "Wireless Sensor Network". 
VII Congresso Brasileiro de Informática na Educação (CBIE 2018)

Anais do XXIX Simpósio Brasileiro de Informática na Educação (SBIE 2018)

\subsection{Bases de Dados de Pesquisa}

As Bases de Dados foram criadas com o propósito de disponibilizar, em uma única plataforma, centenas de revistas científicas e seus respectivos artigos, respaldados por qualidade e originalidade. Para a SLR em questão, as bases escolhidas foram as seguintes: ACM Digital Library, IEEE Xplore, Springer Link e Science Direct. O critério de escolha dessas bases de dados deve-se ao fato de serem as bases mais difundidas no campo da tecnologia. A página do Periódico CAPES também foi consultada, porém apenas servindo de auxílio como motor de busca para as demais bases.

\subsection{Critérios de Inclusão e Exclusão}

De acordo com [Fuzeto and Braga 2016], critérios de inclusão e exclusão são definidos para auxiliar na condução de uma SLR com o intuito de facilitar a classificação de relevância dos estudos. Os critérios de inclusão estão intrinsecamente relacionados à SLR em análise. Dessa forma, ficam definidos como Critérios de Exclusão (CE):

CE1. Todos os estudos que não tratem sobre a relação entre IoT e Educação com Aprendizagem Colaborativa, em casos práticos, serão excluídos;

CE3. Serão excluídos artigos em outros idiomas que não o inglês, devido à baixa predominância de estudos sobre o tema em outros idiomas;

CE3. Caso existam estudos duplicados, apenas um deles será aceito (o mais recente/desenvolvido), excluindo-se todos os demais.

\subsection{Protocolo de Mapeamento}

Na literatura existem várias propostas de protocolos de SLR de forma a ajudar revisores a realizar a leitura de trabalhos selecionados de forma consistente e dentro de intervalos de tempo razoáveis. Dentre os vários modelos existentes, adotou-se o proposto por [Barros and Dwyer 2008] devido à sua capacidade de adaptação às necessidades desta revisão, e às boas referências recebidas sobre. Este modelo orienta uma SLR a partir da realização das seguintes etapas:

1. Selecionar todos os artigos científicos relevantes, relacionados à(s) questão(ões) de pesquisa;

2. Fazer a leitura dos resumos das publicações coletadas e eleger aquelas que satisfazem aos critérios determinados (inclusão/exclusão);

3. Analisar os artigos selecionados na etapa anterior por meio da representação de suas informações através de tabelas e gráficos (de modo opcional, unificar os resultados individuais de cada publicação utilizando técnicas estatísticas).

\section{IoT em CSCL}

Tendo em vista os conceitos de Internet das Coisas e Educação com Aprendizagem Colaborativa, pode-se observar que a união entre elas resulta em uma área de pesquisa e desenvolvimento promissora. Alguns exemplos de tecnologias de IoTSCL, como assim define [Damaševičius and Blažauskas 2017] a utilização de IoT em CSCL, são apresentados a seguir:

- Tipo 1. Redes de sensores/atuadores sem fio: Sensores de câmera, localização, meteorologia, ambiente, presença, movimento, rastreamento ocular, motores e dispositivos servomecânicos, entre outros, que ajudam estudantes na detecção/coleta/compartilhamento de informações e operacionalização de tarefas; 
VII Congresso Brasileiro de Informática na Educação (CBIE 2018)

Anais do XXIX Simpósio Brasileiro de Informática na Educação (SBIE 2018)

- Tipo 2. Tecnologias de comunicação sem fio e de auto-identificação: RFID, NFC, $Q R$ Code e Bluetooth são alguns dos exemplos de tecnologias de IoT que podem auxiliar na identificação e compartilhamento de dados, como também na construção de objetos mais complexos como mesas interativas, robôs educacionais, entre outros. [Puckdeepun and Hussin 2011], por exemplo, utilizaram Bluetooth e infra-vermelho no desenvolvimento de uma mesa interativa compartilhada;

- Tipo 3. Robôs: aparelhos robóticos, de cunho educacional, construídos com auxílio dos mais diversos tipos de sensores e tecnologias de identificação. [Sun and Nishimori 2017] desenvolveram um robô assistente que auxilia o professor na realização de aulas que promovem a colaboração entre alunos, através de jogos que estimulam a competição entre equipes;

- Tipo 4. Mesas e lousas interativas compartilhadas: plataformas touchscreen, que usam desde realidade aumentada até tecnologias IoT (sensores e tecnologias de comunicação e auto-identificação), para a transmissão e recepção de conteúdos educacionais. Um exemplo é o modelo de mesa interativa construída por [Lebrun and Kolski 2015], que utiliza tecnologia de auto-identificação RFID;

- Tipo 5. Equipamentos vestíveis e palpáveis: câmeras e microfones acoplados ao corpo, pulseiras virtuais para ambientes virtuais, luvas inteligentes, entre outros. A tais dispositivos geralmente são incorporados sensores/atuadores que se comunicam de forma autônoma com outros, para a formação de sistemas colaborativos a serem utilizados em ambientes educacionais. [Lechelt and Shum 2016] desenvolveram cubos sensoriais (possuindo uma variedade de sensores, visores de luz e tecnologia Bluetooth) para auxiliar na aprendizagem colaborativa de crianças.

\section{Resultados e Discussão}

Inicialmente, a busca resultou num total de 94 estudos que satisfizeram às especificidades da metodologia aplicada nesta SLR. Entretanto, alguns destes trabalhos não responderam plenamente à sua questão de pesquisa, por: 1) apesar de estarem relacionados a técnicas de utilização de tecnologia para o melhoramento da aprendizagem, não possuíam relação direta com a IoT (17 na área de Interface Homem-Máquina, 11 na de Mobile Learning e 4 na de CSCL); 2) apesar de estarem diretamente relacionados ao conceito de IoT, não tratavam de Aprendizagem Colaborativa (11 trabalhos), e, por fim; 3) apesar de serem pesquisas que envolviam IoT e CSCL, não foram aplicadas em situações reais/práticas (18 trabalhos). Assim, 33 estudos finais foram analisados e serão apresentados a seguir, através de tabela e gráficos ${ }^{1}$.

A Tabela 1 apresenta o número de trabalhos catalogados nas bases de dados pesquisadas. Nela, observa-se que a IEEE possui mais artigos que a soma de todas as outras bases. A Figura 1 apresenta, através da linha de tendência de previsão linear que, apesar de algumas oscilações durante os últimos dez anos, o número de publicações e práticas de IoT em CSCL tende a crescer. É importante ressaltar que o ano de 2018 ainda não se encerrou, mas até o mês de maio (data em que esta SLR foi finalizada) foram catalogados 4 estudos, e que segundo as projeções, pode-se prever que este ano deve encerrar com um número de publicações maior ou igual ao de 2016 (ano com maior incidência de estudos).

As Figuras 2(a) e 2(b) representam, respectivamente, a distribuição destes trabalhos pelos países dos autores/países de publicação (observou-se que apenas um dos

\footnotetext{
${ }^{1}$ A relação dos estudos selecionados encontra-se disponível em: https://sites.google.com/view/iotscl
} 
VII Congresso Brasileiro de Informática na Educação (CBIE 2018)

Anais do XXIX Simpósio Brasileiro de Informática na Educação (SBIE 2018)

\begin{tabular}{|c|c|c|c|}
\hline Bases de dados & Estudos pré-selecionados & Estudos incluídos & \% de estudos incluídos \\
\hline ACM & 17 & 5 & $15 \%$ \\
\hline IEEE Xplore & 53 & 20 & $61 \%$ \\
\hline Springer Link & 17 & 5 & $15 \%$ \\
\hline Science Direct & 5 & 2 & $6 \%$ \\
\hline Outras bases & 2 & 1 & $3 \%$ \\
\hline Total & 94 & 33 & $100 \%$ \\
\hline
\end{tabular}

Tabela 1. Resultado geral nas bases de dados consultadas

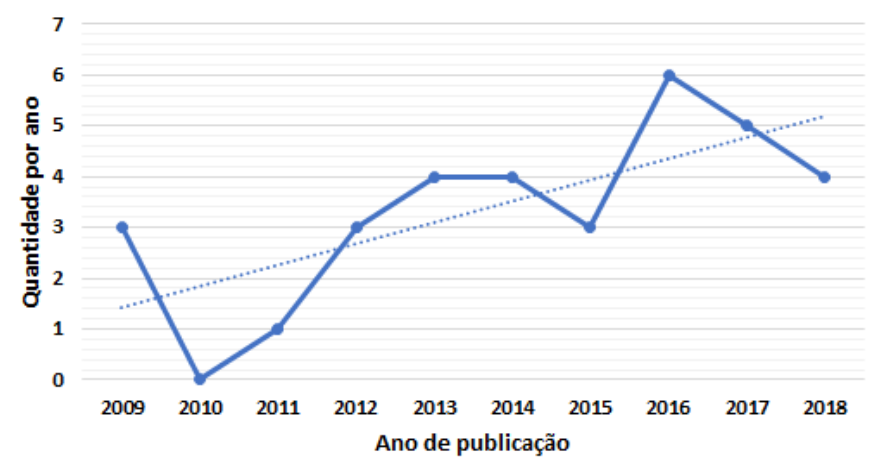

Figura 1. Publicações por ano (com linha de tendência de previsão linear)

trabalhos analisados possui autores pertencentes a 2 países diferentes). Nelas é possível observar que enquanto o Reino Unido possui o maior número de autores (6), é um dos que menos sediou congressos deste domínio de pesquisa nos últimos anos (1). Os Estados Unidos, apesar de possuírem dezenas de pesquisadores trabalhando com IoT em CSCL, tiveram apenas 2 trabalhos publicados que responderam à questão de pequisa proposta nesta revisão, apesar do fato de terem sediado 9 congressos no assunto.

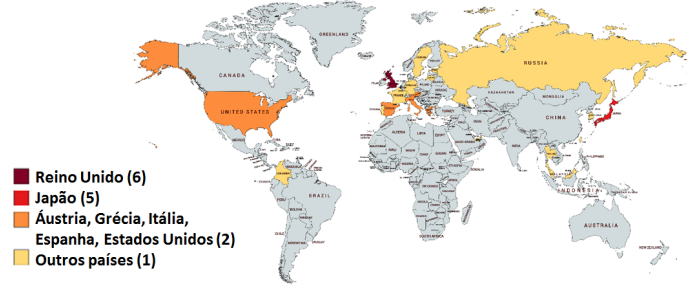

(a)

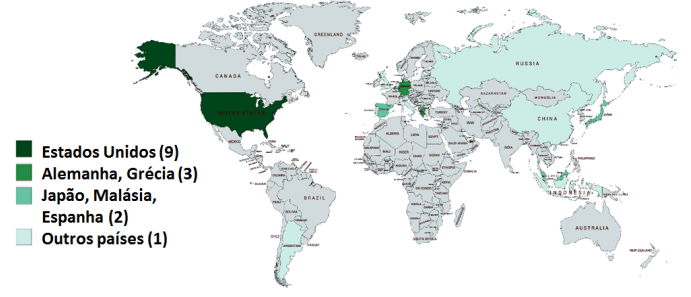

(b)

Figura 2. Distribuição de trabalhos pelos países (a)dos autores/(b)de publicação

A CSCL utiliza técnicas que promovem a colaboração em ambientes de ensino/aprendizagem. Dentre as mais conhecidas, destacam-se a utilização de (I) cenários (ou scripts) e (II) tutores inteligentes. Dos estudos analisados, 60\% utilizaram (I); $9 \%$ (II), e; $31 \%$ não as aplicaram (ou não as informaram). Dos 21 trabalhos que utilizaram (I), a prevalência foi do método "MURDER", com menor incidência para os scripts "Learning Together"e "Jigsaw"(Figura 3). Em alguns trabalhos, os autores realizaram ambas as técnicas em seus experimentos, e em outros, quando utilizando (I), através de uma combinação entre vários cenários (por exemplo, com Jigsaw e MURDER) - em tais situações, os scripts foram considerados e contabilizados jun- 
VII Congresso Brasileiro de Informática na Educação (CBIE 2018)

Anais do XXIX Simpósio Brasileiro de Informática na Educação (SBIE 2018)

tos. Os cenários utilizados nos estudos encontram-se definidos em [Dillenbourg 2002, Dillenbourg and Tchounikine 2007, Hong and Dillenbourg 2008, Hong and Brahm 2009, Karakostas 2009, Kobbe and Fischer 2007, Kollar and Hesse 2006, Stegmann 2004].

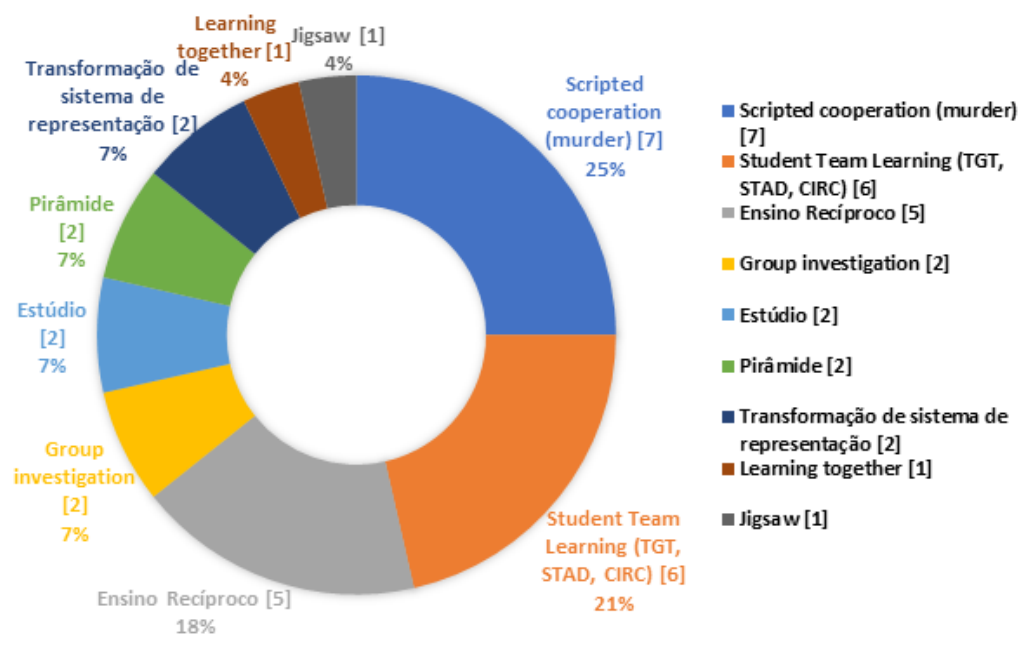

Figura 3. Cenários colaborativos utilizados

Ao focar no nível educacional, extrai-se que a prática de IoT em CSCL foi aplicada em formação geral (treinamentos, cursos de idiomas, etc., com 6 trabalhos) e nos ensinos infantil (4), fundamental (9), médio (9) e superior (17). Levando-se em consideração o número de estudos para cada uma das tecnologias IoT aplicadas em práticas de CSCL, elencadas na seção 3, observa-se 30 experimentos utilizando tecnologias do Tipo 1, 9 do Tipo 2, 6 do Tipo 3, 5 do Tipo 4, e 4 do Tipo 5. Em ambas as análises, observam-se situações em que as práticas são realizadas em mais de um grupo educacional (por exemplo, nos ensinos infantil, fundamental e médio), e/ou utilizando mais de uma tecnologia de IoT (por exemplo, utilizando tecnologias Tipo 1 e Tipo 2). Em tais situações, ambos os grupos educacionais/tecnologias de IoT foram considerados e contabilizados juntos.

Os dados apresentados nos próximos gráficos foram analisados levando-se em consideração mais de duas variáveis, sintetizadas a partir de suas interseções. A Figura 4 apresenta a quantidade e o tipo de tecnologia de IoT empregada por ano de publicação dos trabalhos. Percebe-se que as tecnologias do Tipo 1 lideram em todos os anos (com exceção de 2010, em que não foi encontrado nenhum estudo a respeito), e que aplicações utilizando tecnologias do Tipo 5 são as com menor incidência no período avaliado.

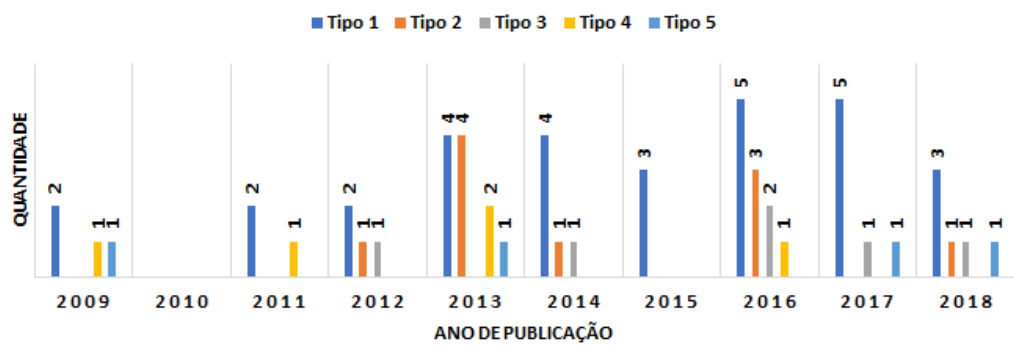

Figura 4. Trabalhos por tipo de tecnologia loT utilizada por ano de publicação 
VII Congresso Brasileiro de Informática na Educação (CBIE 2018)

Anais do XXIX Simpósio Brasileiro de Informática na Educação (SBIE 2018)

A Figura 5 é um gráfico acoplado a uma tabela, e traz consigo a relação entre os grupos educacionais e as tecnologias de IoT utilizadas em contextos de CSCL. Observa-se nesta Figura que tecnologias do Tipo 1 são as mais utilizadas em todos os grupos educacionais, seguidas pelo Tipo 2. Tecnologias Tipo 4 e Tipo 5 possuem resultados semelhantes, sendo os recursos menos aplicados dentre os cinco grupos educacionais analisados.

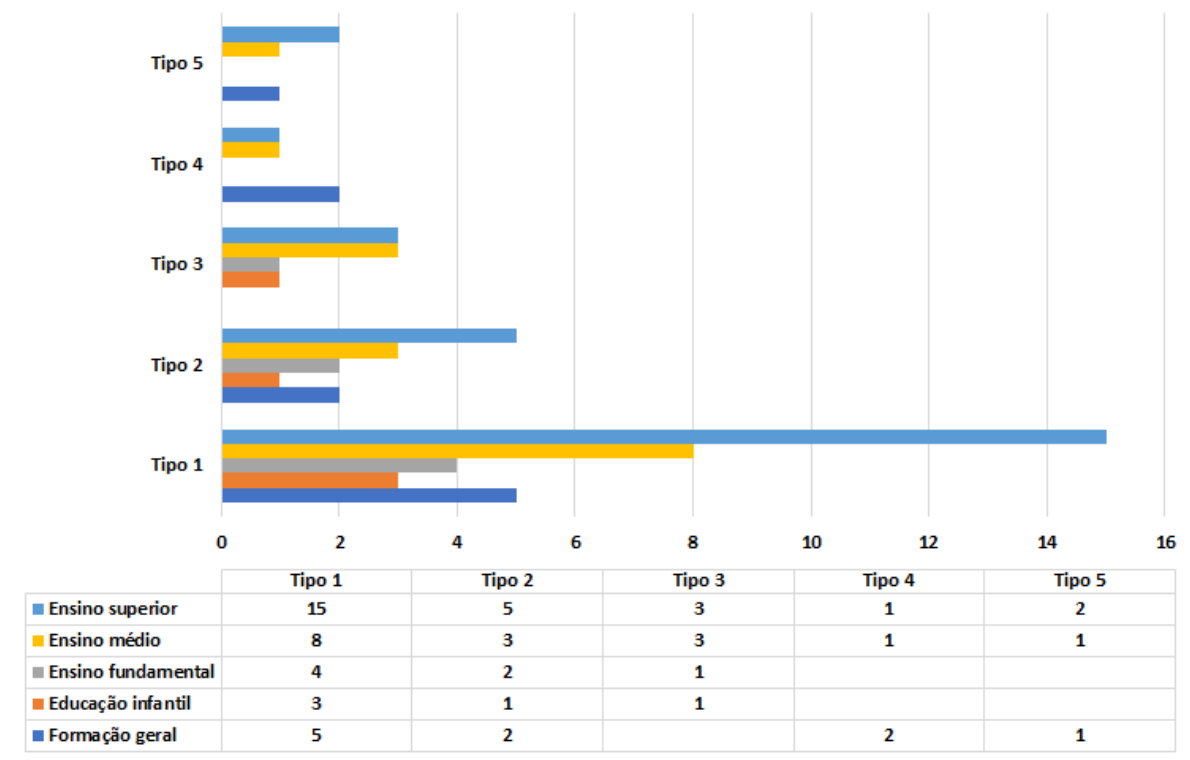

Figura 5. Trabalhos por tipo de tecnologia loT utilizada por grupo educacional

Através de um gráfico do tipo heat chart, encontram-se representados os países dos autores relacionados com a tecnologia de IoT utilizada (Figura 6). Esta Figura apresenta a variação da quantidade de trabalhos em tons de verde, sendo Japão e Reino Unido os países com maior incidência em tecnologias do Tipo 1; Itália, Tipo 2; Japão, Tipo 3; Reino Unido, Tipo 4, e; Alemanha, Grécia, Itália, Rússia e Reino Unido, Tipo 5.

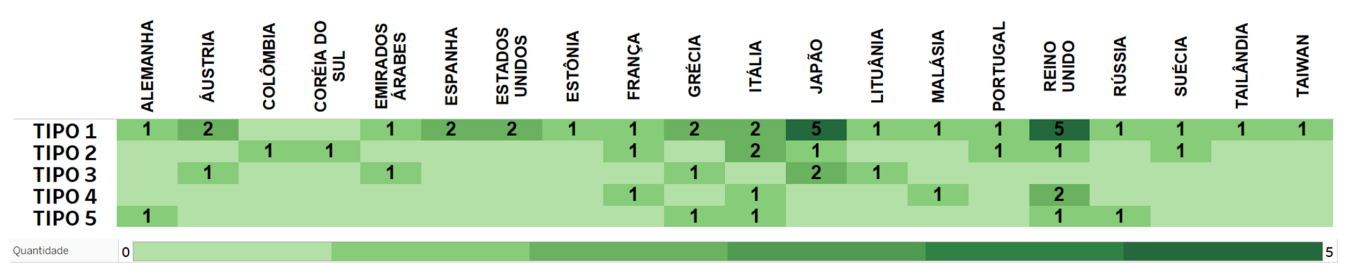

Figura 6. Trabalhos por tecnologias loT utilizadas, por países dos autores

Por fim, a Figura 7 exibe, através de um gráfico de bolhas, a relação entre os países dos autores e os grupos educacionais, mostrando a quantidade da primeira variável em relação à segunda. O Reino Unido lidera tanto no ensino fundamental quanto no superior, com três e dois estudos, respectivamente, empatando em relação ao ensino superior, com Japão e Espanha. Os demais países variam com uma publicação entre os grupos, sendo os Estados Unidos o único país a estar presente em todos os níveis educacionais.

\section{Conclusões e Considerações Finais}

A SLR realizada nesta pesquisa apresenta uma seleção de trabalhos práticos que relacionam a IoT com a CSCL. Dos 33 estudos finais coletados nas quatro bases de dados analisa- 
VII Congresso Brasileiro de Informática na Educação (CBIE 2018)

Anais do XXIX Simpósio Brasileiro de Informática na Educação (SBIE 2018)

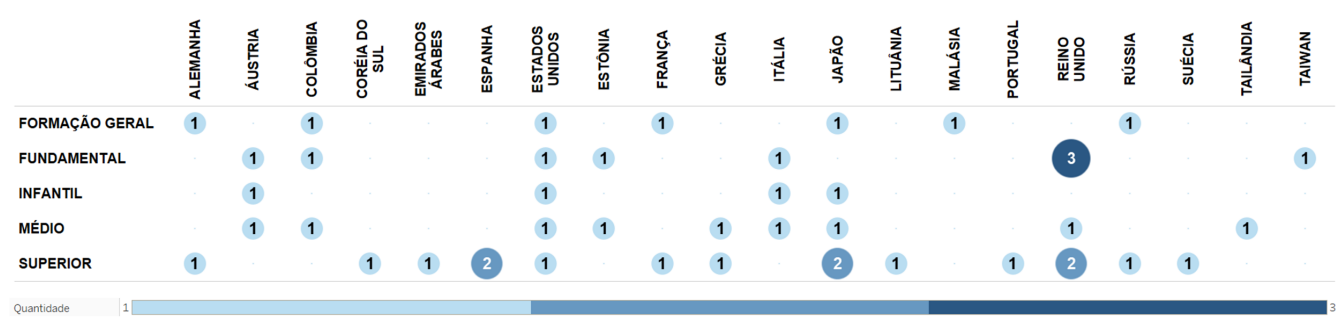

Figura 7. Trabalhos por grupos educacionais, por países dos autores

das, pode-se inferir que entre 2009 e 2018 houve oscilações na quantidade de publicações, mas a tendência é que esses números continuem crescendo, tendo em vista a quantidade de estudos concluídos apenas até maio de 2018.

A Aprendizagem Colaborativa se consolida através de métodos que ajudam os alunos a interagirem de forma eficiente, compartilhando conhecimento. Nos estudos encontrados, observa-se uma maior incidência de publicações utilizando scripts, sendo entre estes o MURDER o mais difundido. O nível educacional em que as práticas de IoT com CSCL foram aplicadas em maior quantidade foi o ensino superior. É curioso perceber que apesar de proporcionar uma gama de oportunidades a serem exploradas, o ensino infantil foi o nível com menor incidência de estudos.

As tecnologias de IoT implantadas pelos autores dos trabalhos contribuíram bastante para o desenvolvimento das práticas colaborativas. As tecnologias do Tipo 1 foram utilizadas em mais de $50 \%$ dos estudos, liderando em todos os grupos educacionais, estando presente em todos os anos do intervalo de pesquisa (com exceção do ano de 2010), e não tendo sido utilizadas apenas nos países Colômbia e Coreia do Sul. As tecnologias do Tipo 2 aparecem em segunda posição neste ranking, abrangendo todos os grupos educacionais e se tornando frequentes nas publicações a partir de 2012. As tecnologias do Tipo 5 foram as menos utilizadas, estando presentes em apenas três dos grupos educacionais analisados (formação geral, ensino médio e superior). Com resultados intermediários estão as tecnologias Tipo 3 (não presentes em cursos de formação geral) e Tipo 4 (não aplicadas nos ensinos infantil e fundamental).

Verificando as publicações pelos países dos autores, percebe-se que o Reino Unido é o local onde as ideias estão sendo colocadas mais em prática, utilizando várias tecnologias de IoT e aplicando diversas técnicas de aprendizagem colaborativa em diferentes grupos educacionais. Japão também é outro país em que essa fusão tem potencial, especialmente no uso de robôs e sensores/atuadores no ensino infantil e superior, através de cenários colaborativos como o MURDER.

Já por país de publicação, os EUA lideram com larga vantagem sobre os demais, o que é um tanto quanto curioso pelo fato de ter colocado em prática apenas alguns poucos trabalhos. Isso pode se justificar, provavelmente, pela maior visibilidade obtida por um artigo ou estudo publicado em meios norte-americanos, em comparação com outros países ainda em desenvolvimento científico/tecnológico. Vale ressaltar também que os Estados Unidos são o único país que aparece em todos os níveis educacionais, enquanto o Reino Unido lidera nos ensinos fundamental e superior.

O potencial da IoT em atuar em vários campos, somado à pluralidade dos métodos 
VII Congresso Brasileiro de Informática na Educação (CBIE 2018)

Anais do XXIX Simpósio Brasileiro de Informática na Educação (SBIE 2018)

de colaboração educacional existentes, representam uma vertente que deve continuar sendo discutida, implementada e aplicada. Entretanto, observando-se o número final de trabalhos desta SLR (33), torna-se possível se pressupor que esta temática ainda se encontra em seus primeiros passos de desenvolvimento, e esta situação pode estar relacionada a vários fatores, como por exemplo:

- A falta de conhecimento dos professores na utilização de novas tecnologias na educação, devido à ausência da discussão destes conteúdos em suas formações;

- A falta de conhecimento das possibilidades que a IoT pode proporcionar à educação, possivelmente devido ao fato que vários professores ainda se encontram praticando a CSCL em salas de aula, presenciais ou a distância, com apenas computadores e/ou celulares, e;

- A falta de conhecimento para implementar cenários colaborativos utilizando tecnologias da IoT, devido ao fato da natureza multidisciplinar necessária ao desenvolvimento de soluções neste domínio (áreas da eletrônica (manipulação de componentes, sensores/atuadores), computação (programação), pedagogia (criação/condução de cenários de aprendizagem), psicologia (cognição, gerência de conflitos), etc., além da própria disciplina objeto de ensino).

Possíveis direcionamentos para a atenuação desta problemática poderiam ser o fomento de grupos multidisciplinares em universidades, dispostos a difundir em centros de ensino a cultura das novas tecnologias na educação colaborativa (IoT em CSCL), e/ou ainda, a inserção dos conceitos do Pensamento Computacional no currículo de crianças e adolescentes, bem como, e principalmente, na formação dos futuros professores.

Por fim, torna-se importante salientar que qualquer pesquisa dessa natureza possui ameaças à validade dos seus resultados. Para este trabalho em particular, detectam-se os seguintes pontos de vulnerabilidade: A pré-seleção dos artigos, devido ao conjunto limitado de bases consultadas, ao número de artigos encontrados, e pelo fato de alguns títulos, resumos e palavras-chave não terem refletido de forma adequada o conteúdo de seus textos; As palavras-chave de busca que, mesmo utilizando vários sinônimos, podem não contemplar a todos os existentes para os termos da pesquisa, podendo ser insuficientes para capturar todos os trabalhos relevantes, e; O longo tempo necessário para a realização de uma SLR, que pode induzir a uma diferente captura de resultados nas bases consultadas, em diferentes datas.

Concluindo-se, esta pesquisa visa trabalhos futuros através do reaproveitamento deste estudo por outros alunos da Universidade Estadual de Santa Cruz, servindo como base para novas revisões e para um trabalho, já em andamento, que utiliza tecnologias de IoT com estudantes do ensino médio para a aprendizagem do conceito de programação. Pretende-se também a continuação desta SLR na busca de novos trabalhos, em novas bases de pesquisa, na tentativa de reduzir as ameaças à sua validade.

\section{Referências}

Barros, A. C.; Wainer, J. C. K. F. L. R. R. and Dwyer, T. (2008). Uso de computadores no Ensino Fundamental e Médio e seus resultados empíricos: uma Revisão Sistemática da Literatura. Revista Brasileira de Informática na Educação, 16(1):57-68.

Damaševičius, R.; Narbutaite, L. P. I. and Blažauskas, T. (2017). Advances in the Use of Educational Robots in Project-Based Teaching. TEM Journal, 6(2):342-348. 
VII Congresso Brasileiro de Informática na Educação (CBIE 2018)

Anais do XXIX Simpósio Brasileiro de Informática na Educação (SBIE 2018)

Dillenbourg, P. (2002). Over-scripting CSCL: The risks of blending collaborative learning with instructional design. pages 61-91. P. A. Kirschner.

Dillenbourg, P. and Tchounikine, P. (2007). Flexibility in macro-scripts for CSCL. Journal of Computer Assisted Learning, 23(1):1-13.

Fuzeto, R. and Braga, R. (2016). Um Mapeamento Sistemático em progresso sobre Internet das Coisas e Educação à Distância. Anais dos Workshops do V Congresso Brasileiro de Informática na Educação (CBIE 2016).

Hong, F. and Dillenbourg, P. (2008). The mechanics of CSCL macro scripts. International Journal of Computer Supported Collaborative Learning, 3(1):5-23.

Hong, F.; Dillenbourg, P. and Brahm, T. (2009). The Manyscripts Pedagogical Handbook: How to build scripts for Collaborative Learning? Universität St. Gallen.

Karakostas, A., D. S. (2009). Adaptation Patterns in Systems for Scripted Collaboration. Int. Conference on Computer-Supported Collaborative Learning, pages 477-481.

Kitchenham, B.; Charters, S. B. D. B. P. T. M. L. S. J. M. M. E. and Visaggio, G. (2007). Guidelines for performing Systematic Literature Reviews in Software Engineering. Technical report. Staffordshire, Reino Unido.

Kobbe, L.; Weinberger, A. D. P. H. A. H. R. H. P. and Fischer, F. (2007). Specifying Computer-Supported Collaboration Scripts. International Journal of Computer Supported Collaborative Learning, 2(3):211-224.

Kollar, I.; Fischer, F. and Hesse, F. W. (2006). Collaboration scripts - a conceptual analysis. Educational Psychology Review, 18(2):159-185.

Lebrun, Y.; Adam, E. M. R. and Kolski, C. (2015). A model for managing interactions between tangible and virtual agents on an rfid interactive tabletop: Case study in traffic simulation. Journal of Computer and System Sciences, 81(3):585-598.

Lechelt, Z.; Rogers, Y. M. N. and Shum, V. (2016). Connectus: A new toolkit for teaching about the Internet of Things. CHI Conference on Human Factors in Computing Systems, pages 3711-3714.

Li, S., Da Xu, L., and Zhao, S. (2018). 5g Internet of Things: A survey. Journal of Industrial Information Integration, 10:1-9.

Mancini, M. (2017). Internet das Coisas: História, conceitos, aplicações e desafios. Disponível em: https://pmisp.org.br/documents/acervo-arquivos/241-internet-das-coisashistoria-conceitos-aplicacoes-e-desafios/file. Acesso em: 27/04/2018.

Puckdeepun, T.; Jaafar, J. H. M. F. and Hussin, F. A. (2011). Investigating collaborative interaction using interactive table and IR devices. Int. Conference on User Science and Engineering, pages 83-88.

Stegmann, K. (2004). Examples of CSCL scripts using of mobile tools. Kaleidoscope Network of Excellence, JEIRP MOSIL. (Deliverable 23.3.1).

Sun, Z.; Li, Z. and Nishimori, T. (2017). Development and assessment of robot teaching assistant in facilitating learning. Int. Conference of Educational Innovation through Technology, pages 165-169. 